\title{
Vibration transfer analysis of component interfaces by a power flow mode approach
}

\author{
Thomas Weisser* — Luc-Olivier Gonidou** \\ Emmanuel Foltête* — Noureddine Bouhaddi* \\ * Département Mécanique Appliquée, Institut FEMTO-ST \\ 24 rue de l'Epitaphe, F-25000 Besançon \\ \{thomas.weisser,emmanuel.foltete, noureddine.bouhaddi\}@univ-fcomte.fr \\ ** Centre National d'Etudes Spatiales \\ Directions des Lanceurs \\ Rond point de l'Espace - Courcouronnes, F-91023 Evry cedex \\ luc.gonidou@cnes.fr
}

\begin{abstract}
A method has been developed to dynamically characterize complex structures' interfaces at low frequencies. The aim is to optimize vibration isolation of a main structure subjected at its junctions to forces generated by connected substructures. An eigenvalue problem is formulated by minimizing the average dissipated power flow of the system. Hence, the derived eigenvalues and eigenvectors describe the energy pattern at each given frequency. It is then possible to characterize the real interface forces and, for example, to control them by determining the appropriated external forces to apply to the structure. This method has been studied on an academic system and applied to a simple coupled structure.

RÉSUMÉ. Une méthode de caractérisation dynamique des interfaces de structures complexes dans le domaine des basses fréquences est présentée. Elle vise à optimiser l'isolation vibratoire d'une structure principale soumise à des efforts de jonction au niveau des liaisons avec différentes sous-structures. En minimisant l'expression de la puissance moyenne dissipée on aboutit à la résolution d'un problème aux valeurs propres. Les valeurs et vecteurs propres ainsi obtenus permettent de décrire la nature des chemins énergétiques en fonction de la fréquence. Il est alors possible de caractériser les efforts réels d'interface et, par exemple, de les contrôler en calculant les forces extérieures appropriées à appliquer à la structure. Cette méthode a été étudiée sur un système académique discret et appliquée à une structure couplée simple.
\end{abstract}

KEYWORDS: vibration transfer, dissipated power-flow, interface forces, substructure.

MOTS-CLÉS : transfert vibratoire, puissance dissipée, efforts d'interface, sous-structure.

DOI:10.3166/EJCM.20.29-47 (c) 2011 Lavoisier, Paris

EJCM - 20/2011. Dynamics of materials, structures and systems, pages 29 to 47 


\section{Introduction}

Industrial structures are often referred to as complex structures. They are composed of an assembly of several substructures, whose mechanical properties generally differ, joined at their interfaces by different junction types. However, their local dynamic behavior may not always be compatible, resulting in difficulties determining the global response of the structure. To analyze and reduce vibration propagation, engineering techniques usually attempt to decouple the substructures by using passive isolators, which tends to be ineffective or even impossible as the complexity of the interface increases. It is therefore necessary to have design methods to perform vibration isolation, i.e. to minimize the vibrational power transmission and to control the power flow through the interface.

The vibration transmission between the boosters and the rocket core of the European space launcher Ariane 5 perfectly illustrates this stake. During atmospheric flight, combustion of the solid propellant excites acoustic modes of the boosters resulting in harmonic oscillations of their structures. One of these modes induces an important dynamic response at the junction with the rocket core, which could have an impact on the dynamic environment. For such reasons, industrials need to have access to design methods to determine optimal power flow paths and thus to ensure vibration isolation (Gonidou, 2007).

Various methods have been developed to solve this kind of problem, whose applicability strongly depends on the considered frequency band and on the complexity of the studied structure. The method presented in this paper is based on power flow analysis (PFA) techniques, which have been first discussed by Goyder and White (Goyder et al., 1980a; Goyder et al., 1980b): their goal is to minimize the power transmission, induced by structural vibrations, between a source and its adjacent structure and then to control the power flow through this structure. By considering a continuous formulation of wave propagation in structures like infinite beams or plates, representing simple machinery foundations, mobility and impedance methods have been introduced to compute the averaged dissipated power (Pinnington et al., 1981). These are derived from the concept of electric impedance (Gardonio et al., 2002) and make the link between the force applied to an element and its dynamic response at a particular observation point. They also have the advantage of allowing substructuring approach: in the case of complex structures each junction can be treated separately and the behavior of the assembled global structure is obtained by means of continuity equations. These methods have then been applied to frame structures by using a direct dynamic-stiffness method (Langley, 1990) and also to simple periodic structures, allowing model size reduction (Cuschieri, 1990).

Based on the equality between the time-averaged power of a system and the energy dissipated by its damping, Miller (Miller et al., 1990) first proposed to perform an eigenvalue analysis of the system's power matrix at the interface to determine frequency ranges and mode combinations which cause the junction to dissipate power. $\mathrm{Su}(\mathrm{Su}$ et al., 1995) hence developed a power flow expression using the eigenmodes 
of the real part of the mobility matrix and derived its upper and lower bounds. These results have been extended and synthesized by $\mathrm{Ji}$ (Ji et al., 2003b; Ji et al., 2003a) in a mobility-based power mode method. To overcome the strong dependency of the mobility matrix on the physical parameters of the system, the frequency and the external force, Xiong (Xiong et al., 2005) have developed a complementary damping-based power flow mode theory and formulated power flow design theorems to control required energy flow dissipation levels and patterns. Other works exposed by Bessac (Bessac et al., 1996), which are complementary based on a primal space formulation, must also be emphasized. Coupling eigenvalues and eigenvectors have been derived by considering a dimensionless coupling matrix, representing the interaction between subsystems. These two quantities allowed to respectively characterize the coupling strength and the modal transmission path between coupled substructures.

This study presents a power flow mode method that comes within a general approach aiming at minimizing the response level of a substructure by minimizing the power flow dissipated at the interfaces with other substructures. This method is based on the eigenanalysis of the interface dynamic flexibility matrix. The derived eigenvalues and eigenvectors respectively represent power flow intensity factors and the associated force distributions or patterns. Particular attention is paid to this set of basis vectors: it does not depend on the external excitations and it spans the power flow space, allowing a complete characterization of the interface forces by projection. The general idea is thus to optimize the junction parameters in order to project the interface forces, generated by a given external loading, onto the power flow subspace that is orthogonal to the most dissipative directions.

Following this introduction, the theoretical formulation of the problem is exposed in Section 2 and the concept of power flow mode is introduced. Then the behavior of the power flow eigenvalues and eigenvectors, with regard to the frequency and to the system's parameters, is described in Section 3. Section 4 details the interface force characterization and develops an optimization procedure to show the dissipated power flow pattern controllability, with regard to the external excitations. Finally, the proposed optimization method is applied to a simple multimode coupled structure in Section 5, before ending with some general conclusions.

\section{Problem statement and formulation}

The general theoretical problem, illustrated Figure 1, can be stated as follows. Let substructure $S_{1}$ be excited by an external force $f_{\text {ext }}$. Interface forces $f_{j}$ are then applied to substructure $S_{2}$ through junctions. This will generate displacements at the interface and a dynamic response $\gamma$ of the whole structure. The purpose of this study is to optimize the structural interface forces $f_{j}$ by minimizing the dissipated power flow at the junction. 


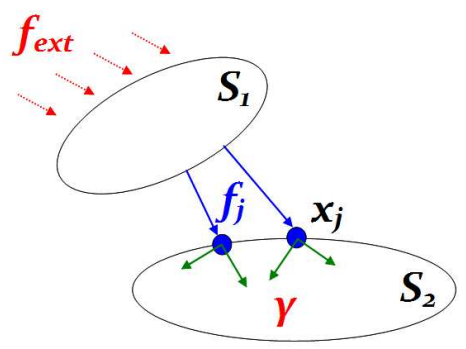

Figure 1. Representation of the interface between two substructures

\subsection{Dissipated power flow of a dynamic system}

The averaged power $\bar{P}$ transmitted in a cycle through a multiple point, $n$ degreeof-freedom (dof), interface subjected to interface forces is given by

$$
\bar{P}=\frac{1}{T} \int_{0}^{T} P_{\text {inst }}(t) \mathrm{d} t=\frac{1}{T} \int_{0}^{T} f_{\text {inst }}(t) v_{\text {inst }}(t) \mathrm{d} t
$$

where $P_{\text {inst }}(t)$ is the instantaneous power, $T$ is the time observation period, $f_{\text {inst }}(t)$ and $v_{\text {inst }}(t)$ are vectors of size $n$ respectively denoting the instantaneous interface force and velocity. By considering a harmonic force of complex amplitude $f$, at a frequency $\omega$, and the resulting velocity of complex amplitude $v$ and after integrating Equation [1] over a single period, the complex power $P_{c}$ can be expressed as (Cremer et al., 2005)

$$
P_{c}(\omega)=\frac{1}{2} f^{*}(\omega) v(\omega)
$$

where the asterisk denotes the transpose complex conjugate quantity. The averaged dissipated power, also known as active power, is then extracted from the real part of the complex power

$$
\overline{P_{\text {diss }}(\omega)}=\Re \mathrm{e}\left(P_{c}(\omega)\right)
$$

As both force and velocity are harmonic, and noticing that $v=j \omega x$, where $x$ is the displacement vector, the averaged dissipated power becomes

$$
\overline{P_{\text {diss }}(\omega)}=\Re \mathrm{e}\left(j \frac{\omega}{2} f^{*} x\right)=-\frac{\omega}{2} \Im \mathrm{m}\left(f^{*} x\right)
$$

In order to express $\overline{P_{d i s s}}$ as a function of the system's parameters, the dynamic flexibility matrix $\Gamma(\omega)$ is derived from the dynamic matrix equation of the associated degrees-of-freedom interface system

$$
\left(-\omega^{2} M+j \omega B+K\right) x=f
$$


where:

- $M$ is the real, symmetric and positive definite mass matrix;

$-K$ is the real, symmetric and semi-positive definite stiffness matrix;

$-C$ is the real, symmetric and semi-positive definite damping matrix;

- $f$ denotes the complex amplitude of the excitation force vector;

such that

$$
x=\Gamma(\omega) f
$$

By substituting Equation [6] into Equation [4] and weighting it by the interface force squared norm, a discretized Rayleigh-like quotient can be derived

$$
Q(\omega)=-\frac{\omega}{2} \Im \mathrm{m}\left(\frac{f^{*} \Gamma f}{f^{*} f}\right)
$$

\subsection{Stationarity of the averaged dissipated power}

The averaged dissipated power formulation given by Equation [7] can be interpreted as the ratio of two quadratic forms and considered as a Rayleigh-like quotient. Thus, optimizing the quotient $Q$ at a given frequency $\omega=\omega_{0}$ comes to differentiating Equation [7] with respect to the real interface force $f=f_{r}$

$$
\frac{\partial Q\left(\omega_{0}\right)}{\partial f_{r}}=0 \Leftrightarrow \frac{\partial s}{\partial f_{r}}=0
$$

According to the stationarity property of the quotient, this leads to solve, at $\omega=\omega_{0}$, the following equivalent eigenproblem

$$
\left(\Gamma_{i}-s_{\nu} I\right) f_{\nu}=0 \quad ; \quad \nu=1, \ldots, n
$$

where $\Gamma_{i}$ is a real matrix of size $n$ denoting the imaginary part of the complex matrix $\Gamma$. It is assumed that $S=\operatorname{diag}\left(s_{\nu}\right)$ is a non-positive diagonal matrix of the power flow eigenvalues $s_{\nu}$ arranged in ascending order, i.e.

$$
s_{1} \leq s_{2} \leq \ldots \leq s_{n}<0
$$

and that $F=\left[f_{1}, \ldots, f_{n}\right]$ represents the orthogonal matrix of the normalized power flow eigenvectors, both satisfying the following orthogonal relations

$$
\begin{aligned}
& F^{T} F=I_{n} \\
& F^{T} \Gamma_{i} F=S
\end{aligned}
$$

where the subscript ${ }^{T}$ denotes the transpose and $I_{n}$ is the identity matrix of size $n$. 
The derived frequency dependent eigenmodes precisely characterize the power flow at the interface: the eigenvalues and eigenvectors respectively give quantitative and qualitative information about the vibration transmission mechanisms at a given frequency. It must also be noticed that these eigenmodes are independent of the external excitations applied to the structure.

\section{Frequency analysis of the power flow eigenproblem}

As stated above, the imaginary part of the dynamic flexibility matrix $\Gamma_{i}$ admits $n$ eigenvalues at a given frequency. However, it depends on the mass, stiffness and damping system's parameters. Thus, a parametric analysis of the power flow eigenproblem is performed. For the sake of simplicity, a two degree-of-freedom academic discrete system will be considered in what follows (see Figure 2). A proportional damping hypothesis, where $C=\alpha K+\beta M$, is initially assumed. The initial values of the system's parameters are summarized in Table 1.

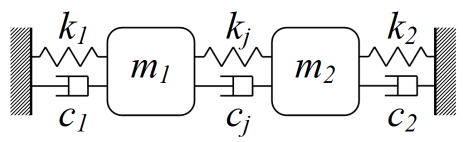

Figure 2. Two dof damped spring-mass system

Table 1. Parameters of the initial system

\begin{tabular}{|c||c|c|}
\hline & Substructure 1 & Substructure 2 \\
\hline Mass $(\mathrm{kg})$ & $m_{1}=2$ & $m_{2}=2$ \\
\hline Stiffness $\left(N . \mathrm{m}^{-1}\right)$ & $k_{1}=1000$ & $k_{2}=1000$ \\
\hline Junction stiffness $\left(N . \mathrm{m}^{-1}\right)$ & \multicolumn{2}{|c|}{$k_{j}=2000$} \\
\hline Proportional damping coefficients & $\alpha=0,001 \quad \beta=0,5$ \\
\hline
\end{tabular}

\subsection{Influence of the system parameters on the power flow eigenvalues}

\section{Case 1: initial analytical study}

At first, the power flow eigenvalues are determined analytically and their behavior is studied with regard to the frequency (see Figure 3). It confirms that both eigenvalues are strictly negative and shows that the two dof system admits two power flow resonances. A complementary analytical study has shown that these are very close but not equal to the dynamic vibration eigenfrequencies of the system, even if damping is taken into account. One should also notice that the curves cross each other: the eigenvalues have been sorted so as to follow their associated eigenvector. In this particular 
case, these are invariant, frequency independent and similar to the dynamic vibration modes.

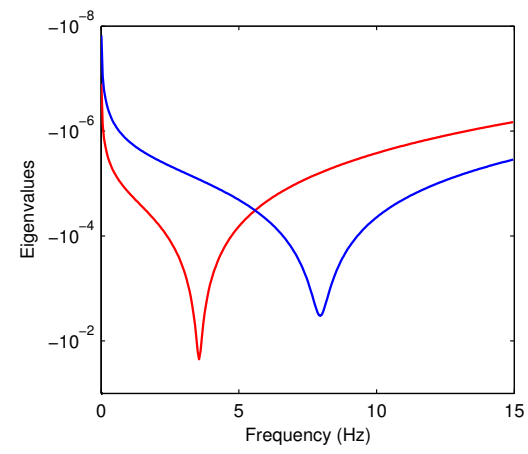

Figure 3. Power flow eigenvalues of the two dof system vs. frequency

\section{Case 2: influence of mass variation}

With regard to the complexity of the analytical expressions in the case of nonsymmetrical system, the parametric study is then carried on numerically. First of all, the influence of the system's mass distribution is investigated. Figure 4 a) presents the variation of the eigenvalues versus frequency for three different values of $m_{1}$ and $m_{2}$, such that $m_{1}=m_{2}$. Their behavior is mainly affected by a shift of the resonance peaks: as the mass increases the power flow resonance frequencies go down and conversely. If the system is modified in a non-symmetric way, i.e. $m_{1} \neq m_{2}$, a frequency shift and a fall of the initially crossing curves around the antiresonance can be observed (see Figure 4 b)).

\section{Case 3: influence of stiffness variation}

The influence of the stiffness parameters is then examined. Whatever the modification, symmetric or not, it implies a frequency shift: as the stiffness increases the power flow resonance frequencies increases too and conversely. It must also be noticed that junction stiffness only acts on the second power flow resonance, which corresponds to the anti-symmetric vibration mode, i.e. the mode where the junction is caused to dissipate power.

\section{Case 4: influence of damping variation}

Finally the damping rate and its model are studied. Initially, the assumption of proportional damping is made, i.e. $C=\alpha K+\beta M$. Thus it is possible to reduce it to modal damping rates. The effect of increasing these rates, illustrated Figure 5 a), results in a smooth decrease of the power flow resonance amplitudes and widths, as encountered with dynamic vibration mode damping. However, by changing this model 


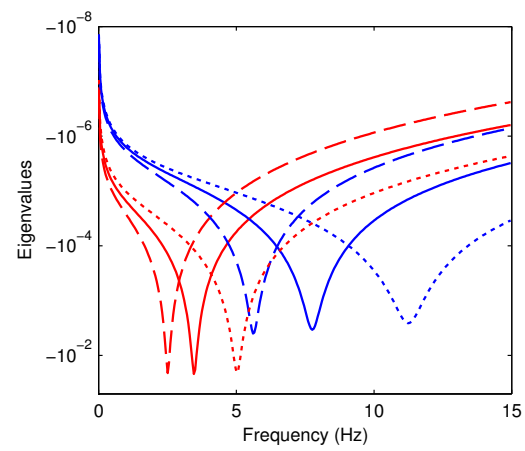

a)

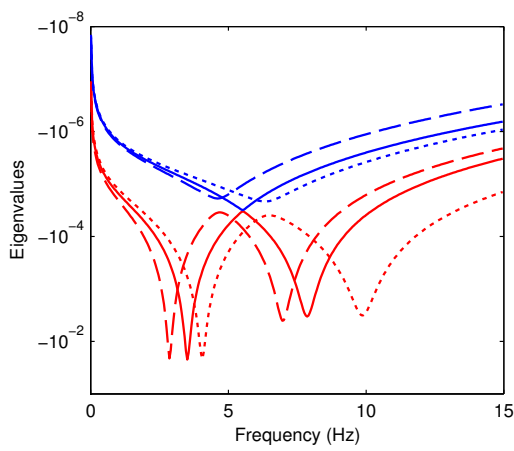

b)

Figure 4. Power flow eigenvalues for different values of a) $m_{1}$ and $\left.m_{2}, b\right) m_{2}$ : full line $+5 \%$; dashed line $+100 \%$; dotted line $-50 \%$

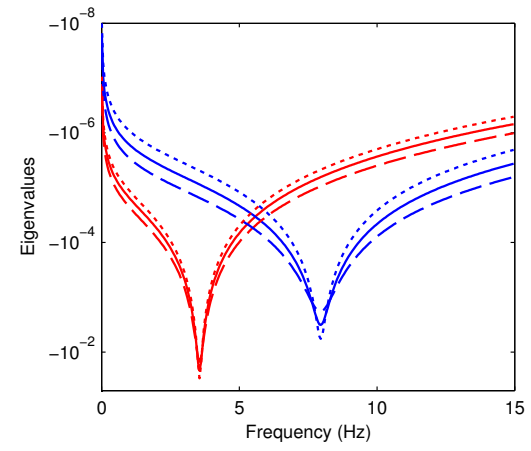

a)

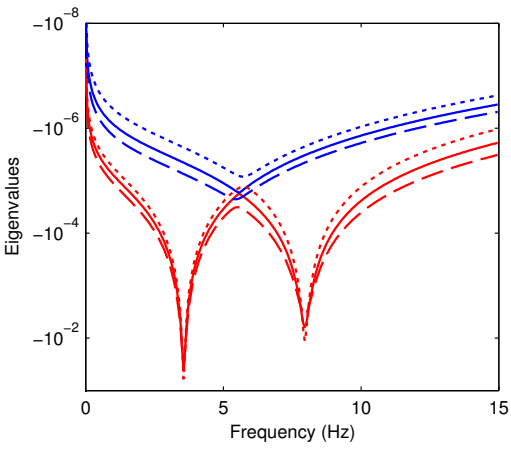

b)

Figure 5. Power flow eigenvalues for different values of a) alpha, b) $c_{2}$ and $c_{3}$ : full line $+5 \%$; dashed line $+100 \%$; dotted line $-50 \%$

into a matrix that does not verify Caughey's condition, i.e. $K M^{-1} C=C M^{-1} K$, a fall the curves is observed again (see Figure 5 b)).

This parametric study has shown that the behavior of the power flow eigenvalues is closely related to the modal one of the vibrating system. However, in the case of independent parameters an interaction between the curves occurs and increases with the perturbation. Both aspects are discussed in the following sections. 


\subsection{Power flow mode determination by a modal approach}

As stated above, the behavior of the power flow eigenvalues with regard to the frequency has shown similarities with the dynamic behavior of the structure. Nevertheless, this direct approach does not allow to derive explicit relationships between the system parameters and its power flow modes. The aim here is to develop a simple expression of these modes depending on dynamic modal parameters.

At first, the conservative natural vibration modes of the system are determined by solving

$$
\left(K-\omega_{\nu}^{2} M\right) y_{\nu}=0 \quad ; \quad \nu=1, \ldots, n
$$

It is stated that $\Lambda=\operatorname{diag}\left(\omega_{\nu}^{2}\right)$ is the spectral matrix containing the $n$ eigenvalues of the structure and that $Y=\left[y_{1}, \ldots, y_{n}\right]$ represents the orthogonal matrix of the associated normalized eigenvectors, both satisfying the following orthogonal relations

$$
Y^{T} M Y=I_{n} \quad Y^{T} K Y=\Lambda \quad Y^{T} B Y=\beta
$$

Assuming that the damping matrix $C$ verifies Caughey's condition (see Section 3.1), $\beta$ becomes a diagonal matrix containing the modal damping rates $\beta_{\nu, \nu}$. The dynamic flexibility matrix is then easily determined and takes the form

$$
\Gamma=Y\left(-\omega^{2} I_{n}+j \omega \beta+\Lambda\right)^{-1} Y^{T}=Y H^{-1}(\omega) Y^{T}
$$

Hence, the equivalent power flow eigenproblem given Equation [12] can be reexpressed

$$
\Gamma_{i}=Y H_{i}^{-1}(\omega) Y^{T}
$$

and finally allows to re-formulate the power flow eigenvalue expression as

$$
F S F^{T}=Y H_{i}^{-1}(\omega) Y^{T} \quad \Rightarrow \quad S=Z H_{i}^{-1} Z^{T}
$$

where $Z=F^{T} Y$. Then, supposing the mass matrix to be proportional to the identity matrix, i.e. $M=m I_{n}$, one finds

$$
Z Z^{T}=\frac{1}{m} I_{n}
$$

Substituting this equation into Equation [17] leads to

$$
S Z=\frac{1}{m} Z H_{i}^{-1}
$$

where both $H_{i}^{-1}$ and $\mathrm{S}$ are diagonal matrices. Consequently, an expression of the power flow eigenvalues is given by the general term of the latter matrix

$$
s_{\nu}(\omega)=-\frac{1}{m} \frac{\beta_{\nu, \nu} \omega}{\left(\omega_{\nu}^{2}-\omega^{2}\right)^{2}+\left(\beta_{\nu, \nu} \omega\right)^{2}}
$$


Furthermore, it is observed that $S Z=Z S$, which means that $Z$ is also a diagonal matrix and that both sets of eigenvectors can be related as follows

$$
Z=F^{T} Y=\frac{1}{\sqrt{m}} I_{n}
$$

By considering the analytical expression given Equation [20] it can be noticed that, whatever the system parameter perturbation, the power flow eigenvalues will always cross each other and admit a single resonance peak (see Figure 3). Numerical simulations have been performed on the previous academic discrete system to compare both direct and modal approaches when the strong hypothesis of Equation [18] is no more verified. Figure 6 a), representing the summed power flow eigenvalues with regard to the frequency, shows that this approach keeps the previously encountered behavior with regard to a mass modification. The relative error between direct and modal approaches can be observed Figure 6 b): it rises as the perturbation, i.e. a nonsymmetric mass modification, increases; reaches its maximum value at the power flow resonance frequency and is equal to zero at the anti-resonance.

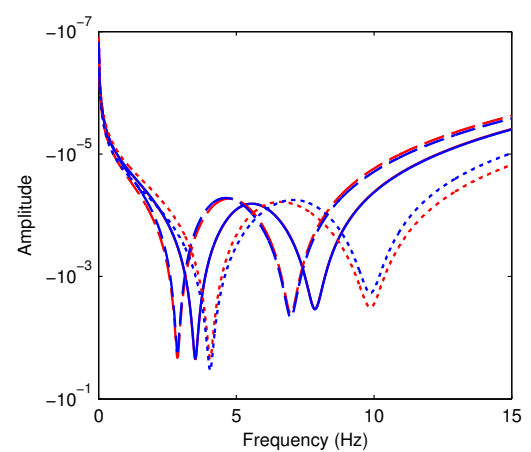

a)

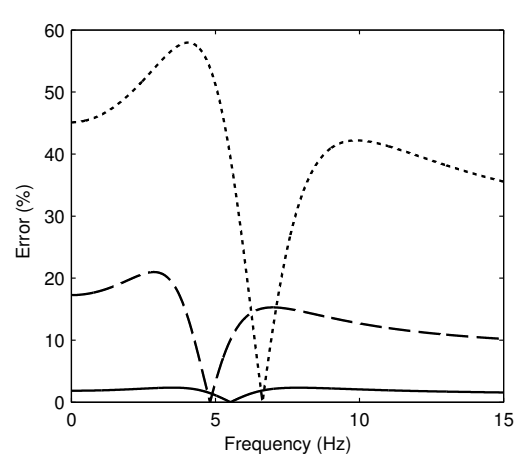

b)

Figure 6. Comparison between direct (black) and modal (grey) approaches for different values of $m_{2}$ : full line $+5 \%$; dashed line $+100 \%$; dotted line $-50 \%$; a) summed eigenvalues; $b$ ) relative error

Further investigations are needed to extend this interesting approach for some general mass matrices and to evaluate its validity from quantitative point of view.

\subsection{Hybridization of the power flow eigenvectors}

Many articles in the literature, e.g. (Pierre, 1988), show that in structures with crossing, double-order eigenvalues, small perturbations of the system's symmetry can compromise this property. These perturbations can generate interactions, sometimes 
in a very localized way, between the eigenvalues, whose loci will come closer until they reach a minimal distance and then diverge. This phenomenon, referred to as eigenvalue loci veering, also implies a hybridization of the associated eigenvectors, whose deformed shapes will be exchanged in a rapid but continuous way. To visualize this, a simple eigenvector veering criterion (EVC) is proposed. It is based on a frequency dependent modal assurance criterion (MAC) and calculated as follows,

$$
E V C_{i j}^{(k, l)}=\frac{\left|f_{k}^{\omega_{i}^{T}} f_{l}^{\omega_{j}}\right|^{2}}{\left|f_{k}^{\omega_{i}}\right|^{2}\left|f_{l}^{\omega_{j}}\right|^{2}}
$$

Figures 7 a) and 7 b) represent the EVC matrix between the first and second power flow eigenvectors at each frequency (from $0 \mathrm{~Hz}$ to $150 \mathrm{~Hz}$ ): orthogonality or collinearity respectively result in a black or white color. On the one hand, the black colored diagonal confirms the fact that power flow eigenvectors constitute a basis for the power flow space at each frequency, even at the power flow resonance, around $6 \mathrm{~Hz}$. On the other hand, the white areas illustrate the exchanged deformed shapes between the eigenvectors. Intermediate colors give information on the quickness and the level of the veering phenomenon. Therefore, as the modal behavior of the system evolves with regard to the frequency, the interface force patterns defined by the power flow eigenvectors hybridize to follow their associated eigenvalues, which are ordered in a decreasing averaged dissipated power flow.

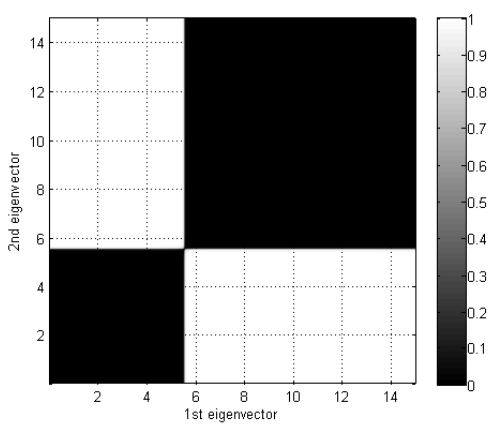

a)

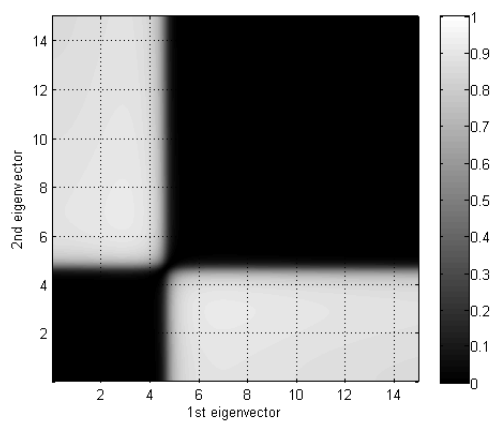

b)

Figure 7. Eigenvector veering criterion for mass $\left.\left.m_{2} a\right)+5 \%, b\right)+100 \%$

\section{Structural interfaces analysis}

The aim of this section is to characterize the vibration transfer between two substructures by means of the power flow mode information previously defined in Section 2 . 


\subsection{Interface forces characterization}

The first step consists in evaluating the forces at each side of the junction, which is supposed to be rigid and non-dissipative in this study. Such junctions are commonly modeled as localized springs of infinite stiffness. The interface forces are thus determined by a substructuring approach

$$
f_{j}=Z_{j i}^{S S_{k}} X_{i}^{S S_{k}}+Z_{j j}^{S S_{k}} X_{j}^{S S_{k}}
$$

where subscripts $i$ and $j$ respectively denote internal and junction dof, $Z^{S S_{k}}$ is the frequency dependent dynamic stiffness matrix of the $k$ th substructure and $X^{S S_{k}}$ its dynamic response.

As exposed in Section 2.2, the force basis $F$ spans the whole power flow pattern space. Hence it is possible to decompose the interface forces as a linear combination into the form

$$
f_{j}=F \alpha=\sum_{\nu=1}^{n} \alpha_{\nu} f_{\nu}
$$

where $\alpha_{\nu}$ are complex coefficients representing the power flow participation of each eigenvector $f_{\nu}$ in $f_{j}$. These are easily determined by means of the orthogonal property of the basis, Equation [11], which yields to

$$
\alpha_{\sigma}=f_{\sigma}^{T} f_{j}=\alpha_{\sigma}^{R}+j \alpha_{\sigma}^{I}
$$

Moreover, by comparing the $\left|\alpha_{\nu}\right|$, a truncated power flow subspace can be constituted to only retain the most significant paths, i.e. $\left[f_{1}, \ldots, f_{m}\right] \subset F$, with $m<<n$. Finally, by substituting these power flow mode parameters into Equation [4], an approximate expression of the averaged dissipated power can be derived. It is related to the optimal interface force configuration, expressed by Equation [24], truncated at the $m$ first eigenmodes and, at a given frequency $\omega_{0}$, is given by

$$
\begin{aligned}
\overline{P^{d i s s}\left(\omega_{0}\right)} & \approx-\frac{\omega_{0}}{2} \sum_{\nu=1}^{m}\left[\left(\alpha_{\nu} f_{\nu}\right)^{*} \Gamma_{i}\left(\alpha_{\nu} f_{\nu}\right)\right]=-\frac{\omega_{0}}{2} \sum_{\nu=1}^{m}\left|\alpha_{\nu}\right|^{2} f_{\nu}^{T} \Gamma_{i} f_{\nu} \\
& =-\frac{\omega_{0}}{2} \sum_{\nu=1}^{m}\left|\alpha_{\nu}\right|^{2} s_{\nu}>0
\end{aligned}
$$

It must be noticed that the obtained averaged dissipated power flow is a positive scalar quantity, contrary to the dissipated energy which may become negative. This expression allows to quantify the truncation error made by only considering the first $m$ power flow modes in the form

$$
0<\varepsilon \frac{\sum_{\nu=1}^{m}\left|\alpha_{\nu}\right|^{2} s_{\nu}}{P^{\text {diss }}\left(\omega_{0}\right)}=\frac{\sum_{\nu=1}^{n}\left|\alpha_{\nu}\right|^{2} s_{\nu}}{\sum^{n}} 1
$$




\subsection{Optimization with regard to the external loading}

The characterization method presented in the previous section can be directly applied in order to optimally control the power flow dissipated at the junction between two substructures. Indeed, by assuming a given junction topology, it is possible to determine, at a given frequency of interest $\omega=\omega_{0}$, the external loading vector which will ca the interface forces to be collinear to an a priori chosen low dissipated power flow pattern.

In this particular case the dynamic equilibrium of the global system is given by

$$
\left\{\begin{array}{l}
Z_{i i}^{S S 1} x_{i}^{S S 1}+Z_{i j}^{S S 1} x_{j}=f_{e x t}^{S S 1} \\
Z_{j i}^{S S 1} x_{i}^{S S 1}+Z_{j j}^{S S 1} x_{j}=f_{j} \\
Z_{i i}^{S S 2} x_{i}^{S S 2}+Z_{i j}^{S S} x_{j}=0 \\
Z_{j i}^{S S 2} x_{i}^{S S 2}+Z_{j j}^{S S 2} x_{j}=-f_{j}
\end{array}\right.
$$

where $f_{\text {ext }}$ is the vector of the external forces applied to substructure $S S 1$. The required power flow pattern is then set by replacing $f_{j}$ with the eigenvector associated to a non dominant power flow eigenvalue. By using a dynamic condensation approach, a matrix expression of $f_{e x t}^{S S 1}$ is derived, which finally comes to solve the following problem

$$
A f_{e x t}^{S S 1}=\overline{f_{j}}
$$

with:

$$
\begin{aligned}
& A=Z_{j i}^{S S 1}\left(Z_{i i}^{S S 1}\right)^{-1} \\
& \overline{f_{j}}=\left[-Z_{j i}^{S S 1}\left(Z_{i i}^{S S 1}\right)^{-1} Z_{i j}^{S S 1}\left(Z_{c}^{S S 2}\right)^{-1}+Z_{j j}^{S S 1}\left(Z_{c}^{S S 2}\right)^{-1}+I_{j}\right] f_{j}
\end{aligned}
$$

It must be noticed that $A$ is a rectangular matrix having a lot more columns than rows, leading to a linear sub-determined inverse problem. In this study, an optimal solution is computed by determining the pseudo inverse matrix $A^{+}$using singular value decomposition technique (Golub et al., 1996).

\section{Application}

\subsection{Model description}

In this section, the power flow mode method is applied to a multimode coupled structure. It is constituted of two clamped-free beams having identical geometric properties but different material parameters. They are also identically discretized (same $2 D$-beam element formulation, same number of nodes) and a proportional damping assumption is made. These two substructures are coupled by means of two complete 
junctions (see Figure 8). The second one is excited at its middle by a constant harmonic force of $10 N$, with a $45^{\circ}$ positioning. A dynamic study of the structure is carried out to determine its natural vibration modes and to compute the dynamic response at the junction nodes (see Figure 9). A frequency band of interest is a priori fixed between $0 \mathrm{~Hz}$ and $500 \mathrm{~Hz}$, which contains ten resonance modes.

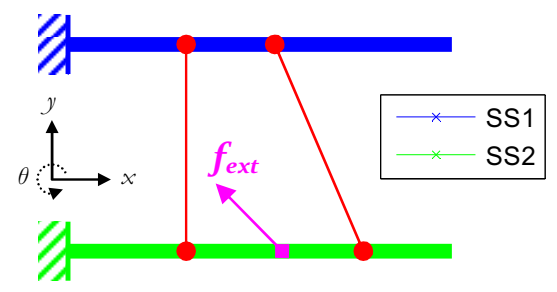

Figure 8. Beams coupled by two rigid junctions on four nodes

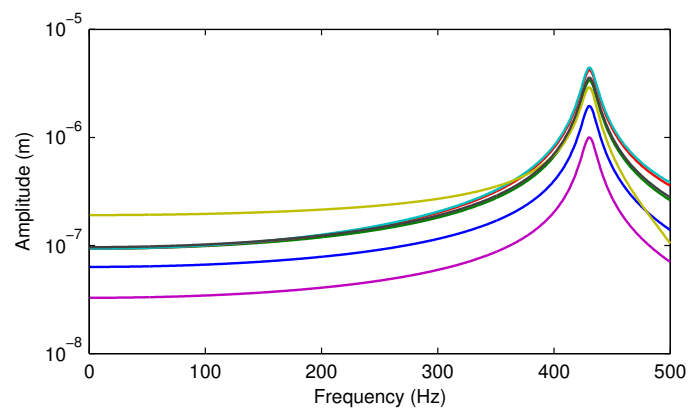

a)

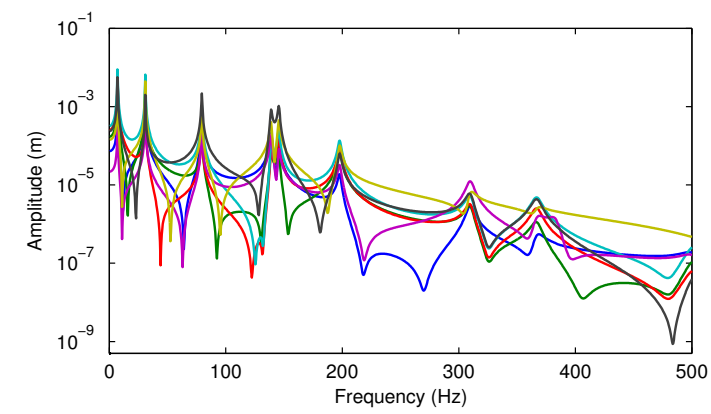

b)

Figure 9. Harmonic response in the a) $x$ direction and b) $y$ direction 


\subsection{Power flow modes}

After having computed the dynamic flexibility matrix and extracted its imaginary part, the power flow eigenproblem is solved at the six interface dof between the two substructures. Figure 10 shows the variation of the eigenvalues versus frequency: these are all negative and ten power flow resonances can be seen at about the same frequencies as natural vibration ones. Both loci veering and crossing can be observed, implying hybridization of the eigenvectors (see Figure 11). The first two eigenvalues represent the major part of the dissipated power flow and are larger than the others, except at $403 \mathrm{~Hz}$ where only the $5^{\text {th }}$ one seems to be sensitive to the first longitudinal vibration mode.

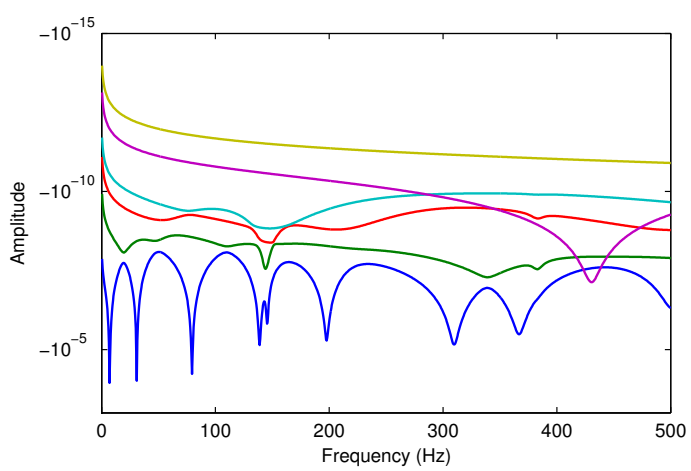

Figure 10. Power flow eigenvalues of the system vs. frequency

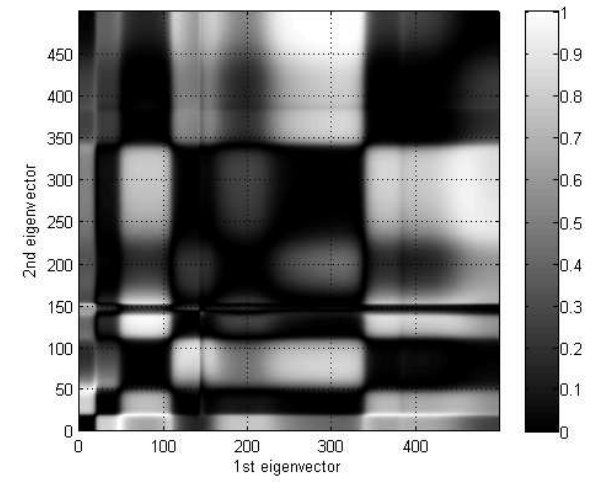

Figure 11. Eigenvector veering criterion for the first two eigenvectors 
As exposed in Section 4.1, the interface forces are then computed and projected on the power flow eigenvector basis. Figure 12 represents the normalized magnitude of the $\alpha_{\nu}$ complex projection coefficients with regard to the frequency. The importance of the first power flow mode is again enlightened around flexural vibration resonances, while the second eigenvector becomes locally predominant at antiresonances. Moreover, the previous remark concerning the longitudinal vibration mode is confirmed by the high value of the $5^{\text {th }}$ projection coefficient between $420 \mathrm{~Hz}$ and $490 \mathrm{~Hz}$.

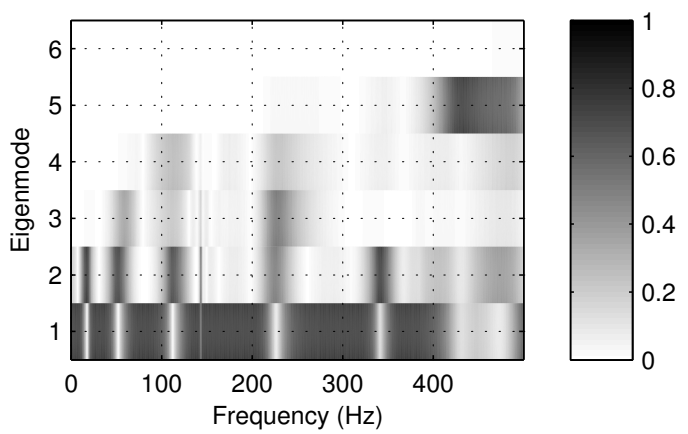

Figure 12. Interface force projection coefficients $\left|\alpha_{\nu}\right|$ vs. frequency

Figure 13 shows the ratio between the total averaged dissipated power flow and the truncated one with regard to the frequency. More than $80 \%$ of the total power flow is achieved by only retaining the first two modes in the projection subspace and the $5^{\text {th }}$ mode is necessary to release the power flow of the longitunal vibration mode.

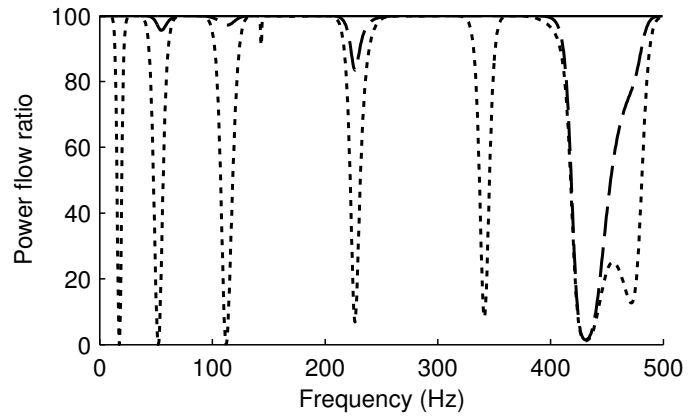

Figure 13. Truncated power flow ratio for: dotted ligne $F=\left[f_{1}\right]$; dashed ligne $F=\left[f_{1}, f_{2}\right]$; full line $F=\left[f_{1}, f_{2}, f_{5}\right]$ 


\subsection{Interface force optimization}

The optimization method exposed in Section 4.2 is finally applied to the coupled beam system. By looking at the harmonic response Figure 9, the energy eigenvalues Figure 10, and the initial projection coefficients Figure 12, it is decided to determine an optimal external force vector so that interface forces are collinear to the $3^{\text {rd }}$ power flow mode, at a frequency of $155 \mathrm{~Hz}$. After solving the inverse problem, a full vector having components of variable magnitude is obtained. It is hence normalized and filtered to only retain the translational dof associated to $80 \%$ of the total norm. The derived force distribution is illustrated Figure 14. It is then applied to the system and the projection coefficients are computed. Figure 15 clearly states that, at $155 \mathrm{~Hz}$, the interface forces are collinear to the $3^{r d}$ power flow mode, inducing a lot less power dissipation than at the inital state (see Figure 12).

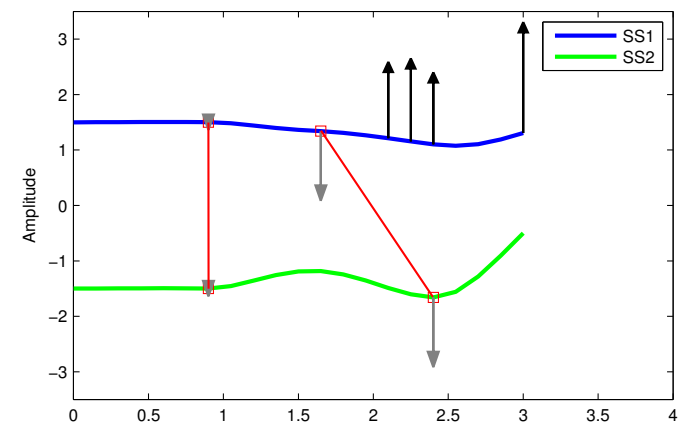

Figure 14. Normalized optimal external force distribution (black), and interface forces (grey)

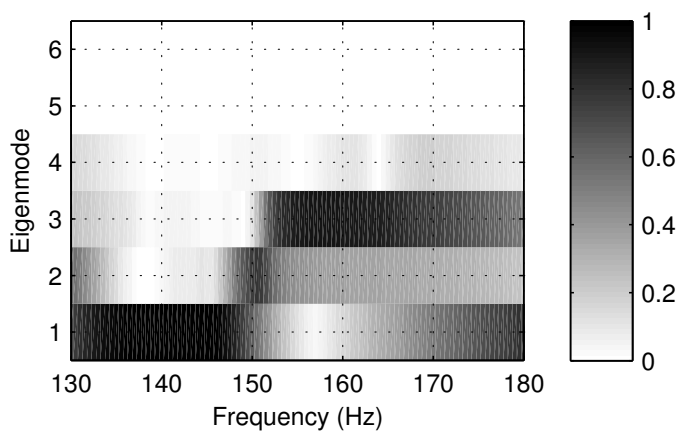

Figure 15. Interface force projection coefficients $\left|\alpha_{\nu}\right|$ vs. frequency 


\section{Conclusion}

A power flow mode method has been developed to characterize the interface forces at the junction between two substructures. It is based on the minimization of the averaged dissipated power flow at the interface, which is equivalent to determining, at each frequency, the eigenmodes of the imaginary part of the junction dynamic flexibility matrix. The derived eigenvalues and eigenvectors are respectively quantitative and qualitative parameters representing the intensity of the dissipated power flow and the pattern of the associated forces. Therefore, it is possible to characterize the interface forces, generated by the external excitations, by projecting them onto the eigenvector basis. An approximated expression of the averaged dissipated power flow can also be obtained by considering a dominant subspace of this basis.

A parametric study has been performed and has shown a complex behavior of the power flow eigenmodes. A modal approach has been proposed to determine a simple analytical expression of the eigenvalues under a strong mass matrix hypothesis. Moreover, eigenvalue loci veering phenomenon has been enlightened and a visualization criterion has been introduced to observe the associated eigenvector hybridization. A simple optimization method has finally been developed and illustrated to show the dissipated power flow pattern controllability, for a given junction topology and at a given frequency, with regard to the external excitations.

On going works concern the extension of the power flow mode method to large complex structures with multiple interfaces, using a component mode synthesis approach. A multi-objective procedure is being studied to determine both optimal and robust junction parameters in order to project the interface forces, generated by a given external loading, onto the power flow subspace that is orthogonal to the most dissipative directions.

\section{Acknowledgements}

This work has been funded by the Future Preparation - Research and Technology Sub-Directorate of the Centre National d'Etudes Spatiales (CNES) - Launchers Directorate (Evry, France).

\section{References}

Bessac F., Gagliardini L., Guyader J.-L., “ Coupling eigenvalues and eigenvectors: a tool for investigating the vibroacoustic behaviour of coupled vibrating systems", Journal of Sound and Vibration, vol. 191, n 5, p. 881 - 899, 1996.

Cremer L., Heckl M., Petersson B., Structure-borne sound, 3rd edn, Springer Verlag, Berlin, 2005.

Cuschieri J., "Vibration transmission through periodic structures using a mobility power flow approach”, Journal of Sound and Vibration, vol. 143, n 1, p. 65 - 74, 1990. 
Gardonio P., Brennan M., " On the origins and developments of mobility and impedance methods in structural dynamics", Journal of Sound and Vibration, vol. 249, n ${ }^{\circ}$ 3, p. 557 - 573, 2002.

Golub G., Loan C. V., Matrix Computations, The Johns Hopkins University Press, third edition, London, U.K., 1996.

Gonidou L.-O., " Dynamic characterization of structural interfaces", Proceedings of the the Spacecraft and Launch Vehicle Dynamic Environments Workshop, 2007.

Goyder H., White R., “ Vibrational power flow from machines into built-up structures, part I: Introduction and approximate analyses of beam and plate-like foundations", Journal of Sound and Vibration, vol. $68, \mathrm{n}^{\circ}$ 1, p. 59 - 75, 1980a.

Goyder H., White R., “ Vibrational power flow from machines into built-up structures, part III: Power flow through isolation systems”, Journal of Sound and Vibration, vol. 68, n ${ }^{\circ}$ 1, p. 97 $-117,1980 \mathrm{~b}$.

Ji L., Mace B., Pinnington R., " Estimation of power transmission to a flexible receiver from a stiff source using a power mode approach", Journal of Sound and Vibration, vol. 268, n 3, p. $525-542,2003$.

Ji L., Mace B., Pinnington R., “ A power mode approach to estimating vibrational power transmitted by multiple sources", Journal of Sound and Vibration, vol. 265, n 2, p. 387 - 399, 2003b.

Langley R., " Analysis of power flow in beams and frameworks using the direct-dynamic stiffness method", Journal of Sound and Vibration, vol. 136, n 3, p. 439 - 452, 1990.

Miller D., Hall S., von Flotow A., " Optimal control of power flow at structural junctions”, Journal of Sound and Vibration, vol. 140, n 3, p. 475 - 497, 1990.

Pierre C., "Mode localization and eigenvalue loci veering phenomena in disordered structures", Journal of Sound and Vibration, vol. 126, n 3, p. 485 - 502, 1988.

Pinnington R., White R., " Power flow through machine isolators to resonant and non-resonant beams", Journal of Sound and Vibration, vol. 75, n² 2, p. 179 - 197, 1981.

Su J., Moorhouse A., Gibbs B., “ Towards a practical characterization for structure-borne sound sources based on mobility techniques", Journal of Sound and Vibration, vol. 185, n ${ }^{\circ}$, p. 737 - 741, 1995.

Xiong Y., Xing J., Price W., “ A power flow mode theory based on a system's damping distribution and power flow design approaches", Proceedings of the royal society, vol. 461, p. 3381 - 3411, 2005. 
